\title{
Lexical and conceptual components of stem completion priming in patients with Alzheimer's disease
}

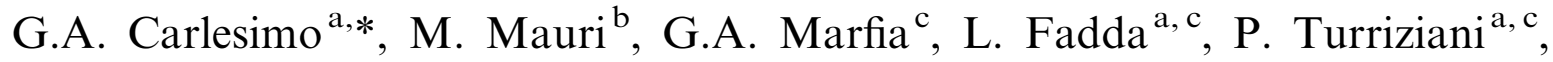 \\ C. Caltagirone ${ }^{\mathrm{a}, \mathrm{c}}$ \\ ${ }^{\mathrm{a}}$ IRCCS S Lucia, V. Ardeatina 306, 00179 Rome, Italy \\ bIRCCS C Mondino, Pavia, Italy

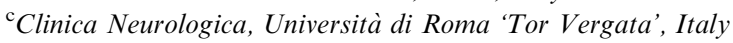

Received 10 December 1997; accepted 9 November 1998

\begin{abstract}
This study evaluated the hypothesis of dissociation between normal lexical but deficient conceptual repetition priming in patients with Alzheimer's disease (AD). For this purpose, we administered to patients with AD and age-matched normal controls the Stem Completion task. In Experiment 1, the level of word processing during study was manipulated by requiring subjects to count vowels (graphemic condition) or generate meanings (semantic condition) of target words. In Experiment 2, the presentation modality was varied during the study to obtain an intramodal and crossmodal repetition priming. Probably due to a floor effect of performance in the graphemic condition, in Experiment 1, AD patients exhibited lower priming than normal controls for the semantically processed words but comparable priming for the graphemically processed ones. In contrast, in Experiment 2, AD patients were poorly primed both in the intra- and crossmodal conditions. Results question the hypothesis of a lexical/conceptual dissociation in the repetition priming exhibited by AD patients and call for other explicative hypotheses of the dissociation between normal and deficient forms of repetition priming in degenerative dementia. (C) 1999 Elsevier Science Ltd. All rights reserved.
\end{abstract}

Keywords: Memory; Repetition priming; Alzheimer's dementia

\section{Introduction}

Impairment in the deliberate recollection of previously experienced facts or information (as revealed by traditional memory tests of Free Recall or Recognition) is the cognitive hallmark of the amnesic syndrome. However, in the last 15 years, a critical advance in the neuropsychological characterization of the amnesic syndrome has been the discovery that patients with a selective disorder of long-term memory are normally primed by previous exposure to visuoverbal or visuo-perceptual stimuli. In particular, it has been demonstrated that when the identification of

\footnotetext{
* Corresponding author. Tel.: 011396 515011; fax: 011396 5032097.

E-mail address: memolab@hsantalucia.it (G.A. Carlesimo)
}

words or pictures presented in a perceptually degraded format, or the completion of fragments from these same stimuli is investigated implicitly, that is, without any explicit reference to their previous presentation, the repetition priming effect (a measure of bias in performance accuracy or speed in favour of previously encountered stimuli) is as large in amnesics as it is in normal age-matched subjects. Following first observations with Stem Completion [19,20], an impressive amount of data was subsequently provided documenting comparable repetition priming in amnesics and age-matched normals in a variety of procedures, such as Identification of tachistoscopically presented words [8], Free Association [45], Lexical Decision about words and non-words [46], and Identification of Fragmented Pictures [49].

Probably the main reason for the neuropsycholo- 
gists' interest in the finding of normal repetition priming in amnesics is that it provides insights about the role of hippocampi and diencephalic nuclei in longterm memory processes. The suggestion that these structures are implicated in the deliberate recollection of previous experiences but that they do not play any role in the repetition priming effect was further qualified by the discovery that patients affected by Alzheimer's disease (AD), who show memory impairment in the explicit domain similar in severity to that exhibited by pure amnesic patients, differ from amnesics in that they frequently show less than normal repetition priming. In 1987, Shimamura and coworkers [44] administered tests of implicit Stem Completion and explicit Word-List learning to groups of AD, Korsakoff's syndrome and Huntington's disease patients. Results demonstrated that while all groups were deficient in both the Free Recall and Recognition of the word-list, only patients with AD exhibited reduced priming in the Stem Completion relative to a group of age-matched controls. In subsequent years, the finding of deficient repetition priming elicited by the Stem Completion task in patients with AD was confirmed by numerous authors $[5,23,43]$ and extended to other repetition priming procedures such as Free Association [5,24,43] and Category Exemplar Generation [35]. In light of this evidence, a neurobiological explanation of the repetition priming-explicit memory dissociation has been proposed based on the different involvement of the mesio-temporal and associative neocortical structures in pure amnesia and degenerative dementia. The involvement of mesio-temporal regions, shared by AD and 'hippocampal' forms of pure amnesia, could underlie the deficient explicit memory common to the two conditions. In contrast, the extension of histopathological changes to associative frontal, parietal and temporal neocortex could be at the base of the impaired repetition priming in patients with $\mathrm{AD}$.

However, evidence regarding poor repetition priming in AD is not unequivocal. In 1991, Keane et al. [28] provided data suggesting a dissociation between normal lexical but deficient conceptual components of verbal repetition priming in degenerative demented patients. ${ }^{1}$ As a predominantly lexical repetition priming task, these authors selected the Word Identification which, in previous studies, was shown to be closely related to modality of presentation [26] but was unaffected by the level of stimulus processing [30]. On this

\footnotetext{
${ }^{1}$ Actually, Keane et al. [28] used the term perceptual as opposed to conceptual priming. However, since perceptual priming in the verbal domain is presumed to reflect the operations of modality specific, presemantic input lexicons, while conceptual priming is the expression of the semantic memory system [47], in this article we prefer using the terms lexical and conceptual priming.
}

task, patients with $\mathrm{AD}$ demonstrated a facilitation effect produced by the previous exposure to stimulus words as large as that exhibited by normal controls. In this same study, Keane et al. [28] used the Stem Completion as a conceptual task and replicated previous results of abnormal repetition priming in patients with AD. Results consistent with those obtained by Keane et al. [28] were successively reported by Gabrieli et al. [16] who described normal priming in patients with $\mathrm{AD}$ in a task involving Identification of Fragmented Pictures (considered as perceptual task) and deficient priming in a Stem Completion task.

In an attempt to account for the proposed dissociation between perceptual and conceptual components of the repetition priming shown by patients with AD, Keane et al. [28] and Gabrieli et al. [16] assumed there is a different neurobiological substrate for the two different forms of priming. In particular, the neural substrate of the presemantic representation subsystems mediating lexical priming (visual input lexicon) and perceptual priming for pictures (structural description system) [47] would be located in the extrastriate cortical regions of the occipital lobes. In contrast, the associative neocortex sustaining the semantic system, assumed to mediate conceptual priming, would be located in more anterior regions of the temporal and parietal lobes. The relative paucity of degenerative changes in the neocortex of the occipital lobes in patients with $\mathrm{AD}$ compared to the diffuse involvement of the associative temporo-parietal neocortex [37] could account for the efficient lexical but deficient conceptual priming in these patients.

The most obvious objection to this view has to do with the nature of repetition priming in the Stem Completion task. As demonstrated by the consistency of the modality effect $[4,6,18]$, repetition priming in Stem Completion certainly has a strong lexical component. In contrast, the contribution of a conceptual component is far less sure. Against the participation of conceptual processes in the rise of priming in Stem Completion is the scarce consistency of the level of processing effect in the literature (for a review see [3]). However, the fact that changing modality from study to testing, despite consistently reducing the size of priming, virtually never abolishes it $[1,19,48]$, supports an additional effect of conceptual on lexical processes in the rise of priming in Stem Completion.

A second concern with Keane et al.'s hypothesis [28] is a methodological one. In fact, if the experimental design involves the comparison of memory tasks that differ in many other respects besides the presumed perceptual or conceptual nature of the priming effect, then we can not be sure whether possible dissociations arising from this comparison are actually the effect of the critical variable of interest, or whether they are expressions of other inter-task differences. It is evident, 
for example, that cognitive operations implied in the identification of tachistoscopically presented words [28] or degraded pictures [16] differ greatly from those necessary to generate a word in response to a graphemic cue. In our opinion, studies investigating the possible role of the level of information processing in predicting memory performance of a pathological group should evaluate performance level on the same memory task following manipulations of encoding or modality. In this way, other variables possibly producing inter-task dissociations (e.g., most convenient cognitive strategy for task solution) would be eliminated and possible functional and/or neuropsychological dissociations arising from this comparison would actually be the expression of the experimental variable of interest (level of information processing).

Finally, the finding of deficient repetition priming elicited by the Stem Completion task in patients with $\mathrm{AD}$ is also not very consistent. In a recent article, Fleischman et al. [14] listed a number of reports documenting comparable priming in patients with $\mathrm{AD}$ and age-matched normal controls. In the authors' opinion, differences in patient selection and/or administration procedure likely accounted for discrepant results. In particular, it is possible that normal or abnormal Stem Completion priming in AD patients actually derives from the adoption of different experimental procedures alternatively stressing the lexical or conceptual contribution to the emergence of repetition priming respectively.

The novelty of the present study is the attempt to disentangle the relative contribution of lexical and conceptual factors in the repetition priming elicited by Stem Completion in patients with AD by manipulating level of processing (Experiment 1) and presentation modality (Experiment 2). In the study phase of Experiment 1, half of the words were analysed according to their graphemic structure (counting vowels) and half were processed at a semantic level (generating meaning). In the second experiment, half of the words were studied auditorily and half visually. During testing, all stems were presented visually, thus giving rise to an intramodal and crossmodal memory condition. The hypothesis of normal lexical but impaired conceptual repetition priming in $\mathrm{AD}$ predicts a dissociation in the implicit memory performance of the demented group as a function of the study condition. In particular, patients with $\mathrm{AD}$ are expected to show particularly abnormal priming when the experimental procedure mainly promotes a conceptual level of stimulus processing. In contrast, when the experimental procedure mainly stresses a lexical level of stimulus analysis, repetition priming in $\mathrm{AD}$ patients is expected to be less or not at all impaired to the extent that a residual conceptual component still contributes to the emergence of priming. As for the Experiment 1, the classical in- terpretation of the advantage of the semantic over the graphemic processing condition in repetition priming is that it reflects the participation of implicit conceptual processes in the rise of priming [42,48]. Accordingly, AD patients are expected to be particularly impaired in the repetition priming elicited by semantically processed words. Priming elicited by graphemically processed words could be partially or also completely spared (thus delineating a relative or an absolute dissociation) to the extent that conceptual processes participate partially or not at all in the processing of words in the graphemic condition. However, not all authors agree with this interpretation of the level of processing effect in implicit Stem Completion. There is evidence, in fact, that the advantage of the semantic over the graphemic condition is actually the result of a contamination of implicit memory processes by explicit retrieval strategies [22] or, alternatively, that it reflects a true implicit priming effect mediated by the more effective processing of lexical (not conceptual) units promoted by the semantic (as opposed to graphemic) orientation task during the study phase [41]. Interpretation of memory processes participating in the rise of priming in Experiment 2 is more straightforward. In fact, while the crossmodal condition surely excludes any participation of perceptual factors in the emergence of repetition priming, the priming elicited by the intramodal condition likely reflects the contribution of both lexical and conceptual levels of stimulus processing. In this case, the dissociation in $\mathrm{AD}$ patients is expected to be relative, with crossmodal priming proportionally more impaired than intramodal priming which, however, is not completely spared to the extent that conceptual processes contribute to the emergence of priming in this condition. As a measure of explicit memory, in both experiments demented and normal controls were also administered the explicit versions of the Stem Completion task.

\section{Experiment 1}

This experiment investigated lexical and conceptual repetition priming in patients with $\mathrm{AD}$ and agematched controls by varying elaborate encoding of the words during the study phase of the Stem Completion task.

\subsection{Material and methods}

\subsubsection{Subjects}

Fifteen patients with AD and 15 age-matched control subjects participated in this study. Demented patients were recruited from the Neurological Clinic of the University of Rome 'Tor Vergata' and from the I.R.C.C.S. 'C. Mondino' in Pavia. All of these patients 
Table 1

Demographic data of patients with dementia and control subjects participating in Experiment 1

\begin{tabular}{llll}
\hline & Age & Years of instruction & Sex $(\mathrm{M} / \mathrm{F})$ \\
\hline AD $(N=15)$ & $67.1(5.9)$ & $6.1(2.5)$ & $5 / 10$ \\
Controls $(N=15)$ & $66.1(6.4)$ & $7.0(3.6)$ & $5 / 10$ \\
\hline
\end{tabular}

had a history of cognitive decline and behavioural changes with normal consciousness. The neuropsychological evaluation documented pathological performances in tasks assessing episodic memory and at least one other cognitive ability (such as visuo-spatial perception, language skills, abstract reasoning). Diagnosis of probable AD was made according to the clinical criteria established by the NINCDS-ADRDA Work Group [33]. Patients were considered to be affected by AD when their Hachinski Ischemia score [21] was three or less (thus highlighting an insidious onset and/ or a progressive deterioration of cognitive deficits and the absence of signs or symptoms suggesting focal brain lesions) and when their neuroradiological examination (cerebral CT scan or MRI) showed cortical and/or subcortical atrophy without signs of focal lesions. Patients with a history of severe head injury, alcoholism or serious and prolonged psychiatric illness were excluded. Finally, all patients underwent laboratory chemistries and EEGs in order to exclude other neurological diseases and major systemic medical illnesses. Dementia severity was mild in eight patients (Clinical Dementia Rating =1 [25]; Mini Mental State Examination $=20.1$ [15]; $\mathrm{SD}=1.9$ ) and mild to moderate in seven patients (Clinical Dementia Rating $=2$; Mini Mental State Examination $=16.4 ; \mathrm{SD}=1.6$ ). In the overall AD sample, the average Mini Mental State Examination score was $19.9(\mathrm{SD}=2.7$; range $=14-23)$.

Control subjects were recruited from the Neurological Clinic of the University of Rome 'Tor Vergata' and from the I.R.C.C.S. 'S. Lucia' in Rome. Some were healthy volunteers. Others were inpatients or outpatients with neurological diseases not affecting the brain (e.g., polyneuritis, spinal trauma, etc.). Patients with AD and control subjects were comparable for age, education and sex distribution (Table 1).

\subsection{Tests and procedures}

\subsubsection{Material}

For both tasks, an implicit and an explicit Stem Completion, 60, 4-11 letters words were selected. These words ranged in frequency of occurrence in Italian from 0 to 131 with a mean of 7.21 per 100.000 [11]. The stem (i.e., the first three letters) of each word was unique among the 60 words and constituted the beginning of at least 10 entries in Zingarelli's
Dictionary [50]. The 60 words were divided into two 30 -word sets in order to create two different test forms. In turn, each 30-word test form was divided into three 10 -word subsets, which were comparable for frequency of occurrence and word length. For each 30word test form, 20 of the words were presented in an initial study phase and in the subsequent stem completion (targets), and the other 10 were presented only in the completion task (foils). Of the 20 words that appeared in the study phase, half were studied in the graphemic condition and half in the semantic condition. The stimuli were counterbalanced across patients in such a way that each 30 -word set was used equally often in the implicit and in the explicit task and each 10-word subset appeared equally often as a target or a foil item and was studied equally often in the graphemic or semantic condition.

\subsubsection{Procedures}

Each patient was administered the implicit task first and then the explicit one. Each task consisted of a study phase, a five-minute interval and a test phase. During the interval, subjects were engaged in performing visuo-spatial tasks.

2.2.2.1. Implicit stem completion. During the study phase, the patients were told that they would see a series of words presented one at a time printed in capital letters in the centre of a sheet of paper. Patients were invited to answer one of two kinds of questions about each word. For half of the words, the experimenter asked them to count the vowels (graphemic condition) and for the other half they were to explain the meaning of the word (semantic condition). Each word was presented for $5 \mathrm{~s}$. The test phase procedure was identical to that in Experiment 1. As a function of the word encoding during the study phase, the words could be divided into three groups of 10 words each: unstudied, graphemically processed, and semantically processed.

2.2.2.2. Explicit stem completion. The procedure was the same as for the implicit task except that at the beginning of the test phase the subject was warned that he/she was going to be presented with a memory task and was asked to complete each stem with words he/she had read in the previous study phase.

\subsection{Results}

As shown in Table 2, both in the implicit and explicit Stem Completion tasks, AD and control subjects completed unstudied words according to the experimental list at the same rate $(t=0.6$ and 1.7 respectively; $P>0.05$ in both cases). This confirmed that the selected set of words was equally familiar to both nor- 
Percentage of explicit and implicit Stem Completion achieved in Experiment 1 by the AD and the control group for unstudied, graphemically and semantically processed words

Implicit stem completion

Unstudied Graphemically processed Semantically processed
Explicit stem completion

Unstudied Graphemically processed Semantically processed

$\mathrm{AD}(N=15)>0.09(0.07)$

$\mathrm{AD}(N=15) \quad 0.09(0.07)$

$0.16(0.08)$

$0.20(0.17)$
$0.19(0.12)$

$0.35(0.12)$
$0.09(0.07)$

$0.04(0.07)$
$0.20(0.10)$

$0.31(0.17)$
$0.24(0.08)$

$0.61(0.15)$ mal and demented groups and allowed us to directly compare absolute priming scores. A preliminary analysis of data revealed a significant learning effect in both groups in all the experimental conditions of Experiment 1. For both the explicit and implicit Stem Completion, in fact, completion of graphemically and semantically processed words consistently surpassed completion of unstudied words (two-tailed $t$-test always $>3.0 ; P<0.05)$.

To directly investigate the effect elicited by different processing conditions on the explicit and implicit tasks, we submitted the learning data (calculated as the difference between the number of graphemically and semantically processed words correctly completed and the number of unstudied words casually completed according to the experimental list) to a three-way ANOVA with Group as between factor and Task (explicit vs implicit) and Condition (graphemically vs semantically processed words) as within factors. The Group effect was highly significant $(F=43.6 ; P<0.001$ with 1,28 df) indicating better overall learning in controls (3.1) than in patients with AD (1.1). The task effect was also significant $(F=24.7 ; P<0.001$ with $1,28 \mathrm{df}$ ) due to the higher completion demonstrated when explicit (2.8) rather than implicit (1.4) retrieval instructions were given. The Condition effect was highly significant $(F=33.5$; $P<0.001$ with $1,28 \mathrm{df}$ ) since many more words were completed following semantic processing (2.7) than graphemic processing (1.5). The Group $\times$ Task interaction was significant $(F=10.3 ; P<0.01$ with $1,28 \mathrm{df})$. In fact, while in normal controls the rate of completion in the explicit test (4.2) was significantly larger than in the implicit one (2.0) $(F=33.4 ; P<0.001$ with $1,28 \mathrm{df})$, the same was not true for patients with AD who completed a similar number of stems with words of the experimental list following implicit (0.9) or explicit (1.3) retrieval instructions $(F=1.5 ; P=$ ns with $1,28 \mathrm{df})$. The Group $\times$ Condition interaction was also significant $(F=18.4 ; P<0.001$ with $1,28 \mathrm{df}$ ). In this case, the interaction was sustained by the fact that while healthy subjects completed significantly more semantically processed $(4.2)$ than graphemically processed (2.0) words $(F=50.8 ; P<0.001$ with $1,28 \mathrm{df})$, stem completion in patients with AD was unaffected by the semantic (1.3) or the graphemic (0.9) level of stimulus analysis $(F=1.1)$. Also the Task $\times$ Condition interaction was significant $(F=5.1 ; P<0.05$ with $1,28 \mathrm{df})$ due to the larger advantage of the semantic over the graphemic condition in the explicit $(+1.7)$ than the implicit $(+0.9)$ test. However, both in the implicit $(F=13.0 ; P=0.01$ with $1,28 \mathrm{df})$ and the explicit $(F=26.5 ; P<0.001$ with $1,28 \mathrm{df})$ task, semantically processed words were completed significantly more than graphemically processed ones. Finally, the three-fold Group $\times$ Task $\times$ Condition interaction approached significance $(F=3.6 ; P=0.06$ with $1,28 \mathrm{df}$ ). Planned comparisons made to qualify this interaction demonstrated that while in the control group the advantage of the semantic over the graphemic condition was much larger in the explicit $(+3.0)$ than in the implicit task $(+1.4)$, in the demented group this advantage was equally negligible both in the explicit $(+0.4)$ and in the implicit $(+0.3)$ tests. Moreover, while in the explicit test healthy controls were better than patients with AD both in the graphemic $(P<0.01)$ and semantic $(P<0.001)$ processing condition, in the implicit test healthy subjects significantly surpassed demented patients in the completion of semantically $(P<0.001)$ but not graphemically $(P>0.10)$ processed words.

In order to exclude that deficient priming in the AD group was actually the effect of too severe cognitive deterioration in our demented patients, we restricted the analysis of results to the comparison of priming scores of the mild AD subgroup $(N=8)$ with those of eight age- and literacy-matched normals. Main results of the three-way ANOVA were largely the same as those involving the whole demented group. In particular, also in this case normal controls were significantly better than patients with $\mathrm{AD}$ in the graphemic (2.6 vs 0.9 ) and semantic (5.8 vs 1.4) condition of the explicit task and in the semantic condition of the implicit task (2.9 vs 0.5). However, in this case, the difference between groups in the implicit completion of graphemically processed words approached significance (2.0 vs $0.6 ; P=0.06$ ).

\subsection{Discussion}

A first finding of this experiment was the sensitivity of implicit Stem Completion to level of processing. In effect, even though in absolute terms the elaborative effect was superior in the explicit than in the implicit 
Stem Completion (significance of the Task $\times$ Condition interaction), in proportional terms the advantage of the semantic over the graphemic condition was about the same (0.90) following both implicit and explicit retrieval instructions. Data consistent with a positive effect of the semantic elaboration on the repetition priming elicited by the Stem Completion have been reported by Bowers and Schacter [2], Graf and Mandler [17] and Lupker et al. [31] among others. However, Chiarello and Hoyer [9], Java and Gardiner [27] and Micco and Masson [34] found the same level of priming in Stem Completion irrespective of graphemic or semantic level of stimulus analysis.

In the present experiment, demented patients did not differ from controls in the graphemic condition of the implicit Stem Completion, but completed semantically processed words significantly less than controls. According to the prevalent opinion that the level of processing effect in implicit Stem Completion reflects the contribution of implicit conceptual processes in the rise of priming [42,48], these data are consistent with the hypothesis of a dissociation between normal lexical but deficient conceptual repetition priming in $\mathrm{AD}$ patients. There are, however, both empirical and theoretical problems with this interpretation. From an empirical point of view, a note of caution is suggested by the very low priming achieved by normal controls for the graphemically processed words (1.3). In effect, although the priming effect obtained by patients with $\mathrm{AD}$ in this experimental condition was lower than that displayed by normal controls, a floor effect of performance could have prevented a significant statistical difference between the groups from emerging. Supporting this suggestion, the mild AD subgroup showed a trend toward deficient priming also for the graphemically processed words, probably due to the higher repetition priming achieved in this experimental condition by the age-matched normal subjects (2.0) relative to the overall control group. From a theoretical perspective, there is no general agreement that the larger priming elicited by semantic processing over graphemic processing in Stem Completion actually reflects the contribution of implicit conceptual processes to priming. At least two alternative accounts for this effect have been proposed. Based on evidence of a significant elaborative effect in healthy but not in amnesic patients, Hamman and Squire [22] proposed reliance on explicit retrieval strategies as a possible reason for the significant level of processing effect in Stem Completion. However, against such an interpretation, Carlesimo [4] and Graf et al. [20] reported the same advantage of the semantic over the graphemic condition in the Stem Completion priming exhibited by normals and by amnesic patients. Moreover, Richardson-Klavehn and Gardiner [41] recently reported convincing data supporting the hypothesis that the level of processing effect in implicit Stem
Completion does not reflect the contamination of the implicit test by explicit retrieval strategies or the contribution of conceptual processes to the emergence of repetition priming but, rather, is the expression of a lexical level of stimulus processing. In their study, in fact, a graphemic processing condition similar to that utilized in the present study (i.e., counting letters containing enclosed spaces) elicited significantly less priming than a phonological processing condition (counting syllables in words) and a semantic processing condition (rating pleasantness of words) which, in turn, gave rise to similar priming. These authors suggested that just focusing attention in isolated letters does not mean that words are processed as lexical units. In contrast, counting syllables or rating pleasantness promotes a lexical level of word analysis, thus accounting for larger priming in these conditions than in the graphemic processing condition. Finally, the fact that the semantic processing condition did not provide any further advantage over the phonological processing one strongly suggests that the significant level of processing effect in implicit Stem Completion reflects a lexical rather than a conceptual level of stimulus analysis. It is worth noting that in a parallel explicit Stem Completion of the same study, the canonical level of processing effect, with semantically processed words better remembered than phonologically processed ones, was described.

Following this line of reasoning, the larger priming elicited in the normal subjects of the present experiment by semantically than graphemically processed words should be interpreted as the result of more effective lexical processing in the earlier than in the later processing condition. In the same vein, the very low priming elicited by the graphemically processed words (likely responsible for the lack of difference between normals and AD patients) could be the expression of insufficient lexical processing promoted by this kind of stimulus analysis.

The second experiment provided the opportunity to more appropriately test the question of spared lexical but deficient conceptual repetition priming in $\mathrm{AD}$ patients. In contrast with uncertainties characterizing the interpretation of the level of processing effect in repetition priming, there is general agreement that manipulating presentation modalities from study to test provides an effective tool for discriminating the contribution of lexical and conceptual processes to the rise of verbal repetition priming. In particular, while changing modality from study to testing ensures that perceptual processes do not contribute to priming and that all the observed priming rises from conceptual processes, maintaining the same modality during study and testing elicits a priming that is the result of a contribution of both perceptual and conceptual processes. Accordingly, if the hypothesis of normal lexical prim- 
Table 3

Demographic data of patients with dementia and control subjects participating in Experiment 2

\begin{tabular}{llll}
\hline & Age & Years of instruction & Sex $(\mathrm{M} / \mathrm{F})$ \\
\hline AD $(N=12)$ & $67.2(6.2)$ & $6.4(2.5)$ & $5 / 7$ \\
Controls $(N=12)$ & $70.3(7.8)$ & $7.6(3.6)$ & $5 / 7$ \\
\hline
\end{tabular}

ing in patients with $\mathrm{AD}$ is correct, then these patients are expected to be proportionally more impaired in crossmodal priming (exclusively based on a conceptual level of stimulus analysis) than in intramodal priming (at least partially based on a lexical level of stimulus analysis). A similar or also a larger advantage of intramodal over crossmodal priming in the AD group compared to healthy subjects is also expected.

\section{Experiment 2}

In Experiment 2, we manipulated the presentation modality in order to obtain intramodal and crossmodal retrieval conditions in both the implicit and explicit versions of the Stem Completion task.

\subsection{Material and methods}

\subsubsection{Subjects}

Twelve patients with $\mathrm{AD}$ and 12 normal controls recruited following the same criteria of Experiment 1 participated in this experiment. Two patients had already participated in Experiment 1 . In this case, dementia severity was mild in nine patients (Clinical Dementia Rating=1; Mini Mental State Examination $=20.8 ; \mathrm{SD}=1.4)$ and mild to moderate in 4 patients (Clinical Dementia Rating $=2$; Mini Mental State Examination $=15.3 ; \mathrm{SD}=1.3$ ). In the overall $\mathrm{AD}$ sample, the average Mini Mental State Examination score was $19.1(\mathrm{SD}=3.0$; range $=14-23)$. Demented and normal subjects were similar for age, literacy and sex distribution (Table 3).

\subsubsection{Material}

For this experiment, another 60, 5-12 letters words were selected. Criteria followed to select and divide words into six 10-word subsets were the same as those already described in Experiment 1.

\subsubsection{Procedures}

Each patient was administered the implicit task first and then the explicit one.

3.1.3.1. Implicit stem completion. During the study phase, patients were told that they would see or hear a series of words. In the visual condition, words printed in capital letters in the centre of a sheet of paper were presented for $5 \mathrm{~s}$. In the auditory condition, words were read by the examiner. The 10 words presented visually and the ten words presented auditorily were administered alternatively. The patients were requested to rate how much they liked or disliked each word, whether presented visually or auditorily, on a 5-point scale. The experimenter waited for the patient to rate each word before proceeding to the next. The study phase was followed by a five minute interval and then by the test phase. During this phase, 30 three-letter word stems were presented one at a time in the visual modality. Patients were asked to complete each stem with the first word that came to mind. As a function of the modality of presentation during the study phase, the words could be divided into three groups of 10 words each: unstudied, visually presented and auditorily presented.

3.1.3.2. Explicit stem completion. The only difference between this test and the previous one was that at the beginning of the test phase the examiner told the subject that he was going to be presented with a memory task and asked him/her to complete each stem with words studied in the previous phase.

\subsection{Results}

Both in the implicit and explicit Stem Completion tasks, AD and control subjects completed unstudied words according to the experimental list at the same rate $(t=0.1$ and 0.5 respectively; $P>0.05$ in both cases). A preliminary analysis of absolute completion scores (Table 4) demonstrated a significant memory effect in the control group in all the conditions of Experiment 2. In fact, in both the explicit and implicit tasks, completion of visually and auditorily studied words was significantly higher than completion of unstudied words (two-tailed $t$-test consistently $>3.8$; $P<0.05$ ). Patients with $\mathrm{AD}$, instead, completed significantly more visually and auditorily studied that unstudied words in the implicit test $(t>3.1 ; P<0.05)$ and more visually studied than unstudied words in the explicit test $(t=2.7 ; P<0.05)$. The completion of auditorily studied words in the explicit test, instead, was not significantly elevated over chance $(t=1.6 ; P>0.10)$.

To directly investigate the modality effect in the implicit and explicit completion tasks, we compared levels of learning evidenced by the two groups of patients in the intramodal and crossmodal conditions. As a measure of learning (both explicit and implicit), for each patient we calculated the difference between the number of visually and auditorily studied words correctly completed and the number of unstudied words casually completed according to the experimental list. These data were analysed by a three-way 
Percentage of explicit and implicit Stem Completion achieved in Experiment 2 by the AD and the control group for unstudied, auditorily and visually presented words

\begin{tabular}{|c|c|c|c|c|c|c|}
\hline & \multicolumn{3}{|c|}{ Implicit stem completion } & \multicolumn{3}{|c|}{ Explicit stem completion } \\
\hline & Unstudied & Auditorily presented & Visually presented & Unstudied & Auditorily presented & Visually presented \\
\hline $\operatorname{AD}(N=12)$ & $0.15(0.10)$ & $0.25(0.12)$ & $0.25(0.13)$ & $0.18(0.14)$ & $0.26(0.12)$ & $0.32(0.17)$ \\
\hline Controls $(N=12)$ & $0.14(0.13)$ & $0.36(0.14)$ & $0.49(0.19)$ & $0.15(0.14)$ & $0.52(0.11)$ & $0.77(0.11)$ \\
\hline
\end{tabular}

ANOVA with Group as between factor and Task (explicit vs implicit) and Modality (visually vs auditorily studied) as within factors. Results of this analysis revealed significantly larger learning in the Control (3.9) than in the AD group (1.1) $(F=33.4 ; P<0.001$ with $1,22 \mathrm{df})$. The task effect was also significant $(F=5.8$; $P<0.05$ with $1,22 \mathrm{df}$ ), due to greater completion in the explicit (3.0) than in the implicit (1.9) test. Finally, the Modality effect was significant $(F=21.1 ; P<0.001$ with $1,22 \mathrm{df}$ ) with visually studied words (3.1) completed better than auditorily studied words (1.9). The Group $\times$ Task interaction was significant $(F=5.1 ; P<0.05$ with $1,22 \mathrm{df})$. In fact, while control subjects completed significantly more stems with words of the studied list in the explicit (5.0) than in the implicit (2.8) condition $(F=10.5 ; P<0.01$ with $1,22 \mathrm{df})$, patients with AD completed a similar number of stems in the explicit (1.1) and implicit (1.0) tests $(F=0.01)$. The Group $\times$ Condition interaction was also highly significant $(F=11.2 ; P<0.01$ with $1,22 \mathrm{df}$ ) due to the larger completion in the intramodal (4.9) than in the crossmodal (2.9) experimental condition in the control group $(F=30.2 ; P<0.001$ with $1,22 \mathrm{df})$, but not in the demented group ( 1.2 vs $0.9 ; F=0.8)$. The marginally significant Task $\times$ Condition interaction $(F=4.6 ; P<0.05$ with 1,22 df) reflected the larger advantage of the intramodal (3.8) over the crossmodal (1.6) condition in the explicit Cued Recall $(F=34.8 ; P<0.001$ with $1,22 \mathrm{df})$ than in the implicit Stem Completion (2.3 vs 1.6) $(F=3.8 ; P=0.06$ with $1,22 \mathrm{df})$. Finally, the threefold Group $\times$ Task $\times$ Condition interaction was not significant $(F=1.0)$. In fact, patients with $\mathrm{AD}$ were poorer than normal controls in all the experimental conditions (intra- and cross-modal completion of both the implicit and explicit tests) ( $P$ consistently $<0.01$ ).

The possible role of severity of dementia in the emergence of deficient repetition priming in patients with $\mathrm{AD}$ was assessed by re-evaluating results only for the mild AD subsample $(N=9)$. Results largely replicated findings for the overall sample. In particular, patients with $\mathrm{AD}$ were significantly worse than normal controls both in the intramodal (4.3 vs 1.1 ) and crossmodal (2.1 vs 0.9$)$ priming and in the intramodal (6.2 vs 1.4 ) and crossmodal (3.6 vs 0.6 ) explicit completion.

\subsection{Discussion}

Confirming previous data from our laboratory [6], in the control group both the implicit and explicit Stem Completion were sensitive to modality of stimulus presentation. In both cases, in fact, significantly more stems were completed with previously studied words when the same modality of presentation (visual) was used during study and test than when it mismatched from study (auditory) to test (visual). In this case, the advantage of the intramodal over the crossmodal experimental condition was larger in the explicit than in the implicit task also when expressed in proportional terms (0.66 vs 0.44$)$.

Consistent with the view of a significant conceptual component in the repetition priming elicited by Stem Completion, the crossmodal condition also produced a significant priming effect in both normal and pathological groups (for consistent results, see $[1,19,48]$ ).

Results of Experiment 2 were in striking contrast with predictions of normal lexical but deficient conceptual repetition priming in patients with AD. Contrary to expectations, in fact, our demented group did not demonstrate any modality effect in the implicit Stem Completion. Priming levels in patients with AD were roughly both the same both when presentation modalities during study and test matched and when they mismatched. Moreover, with respect to the normal group, AD patients' repetition priming was relatively more impaired in the intramodal than in the crossmodal experimental condition.

\section{General discussion}

This study was aimed at evaluating previous suggestions about a dissociation between normal perceptual but deficient conceptual repetition priming in patients with AD. This hypothesis was advanced by Keane et al. [28,29] and Gabrieli et al. [16] to account for the evidence in degenerative demented patients of normal repetition priming in predominantly perceptual tests (Identification of tachistoscopically presented words and non-words and Identification of fragmented pictures) and deficient priming in Stem Completion, con- 
sidered at least partially subsumed by conceptual processes. The hypothesis made good sense since semantic knowledge (presumed to mediate conceptual priming) is known to be early impaired in the progress of the disease [39], while lexical and visuo-perceptual functions (underlying perceptual priming) are less precociously compromised. Keane et al. [28] also suggested a neuropathological account for this discrepancy in that degenerative changes in $\mathrm{AD}$ very diffusely affect associative neocortex in temporal and parietal lobes (presumed to underlie the semantic system), while extrastriate cortex of the occipital lobes (the neural substrate of the perceptual representation subsystems) is much less involved [37]. Fleischmann et al. [13,14] recently interpreted in a similar vein the finding of normal Word Identification and Stem Completion repetition priming in $\mathrm{AD}$ patients following both reading and generating critical items during the study phase. Based on the observation of deficient performance of their AD patients on a variety of semantic memory tests, Fleischmann et al. [14] concluded that normal priming in their patients "may reflect non-semantic changes in the availability of lexical entries interacting with normal perceptual processes" (p. 31). Overall, our results did not confirm this hypothesis. Although the results of Experiment 1, documenting normal priming in patients with $\mathrm{AD}$ for the graphemically processed words but deficient priming for the semantically processed ones, were consistent with the lexical-conceptual dissociation hypothesis (but see the Discussion section of Experiment 1 for theoretical problems with interpreting the level of processing effect in implicit Stem Completion as reflecting the contribution of conceptual factors to priming), a closer scrutiny of these results suggests that a floor effect of performance could have prevented pathological priming from emerging for the graphemically processed words in patients with AD. Results of Experiment 2 are straightforward in confirming this. In fact, patients with $\mathrm{AD}$ were poorly primed in both the intramodal and crossmodal task conditions. Most importantly, they did not exhibit any facilitation due to the physical congruity between stimulus at study and fragment cue at test. In fact, level of priming was substantially the same when the same or different presentation modalities were used during study and testing. In conclusion, results of the present study point out a general decrement of Stem Completion priming in $\mathrm{AD}$ patients, encompassing both the lexical component (lack of an advantage of the intramodal over the crossmodal condition in Experiment 2 and of the semantic over the graphemic condition in Experiment 1) and the conceptual component (deficient crossmodal priming in Experiment 2).

Experimental evidence regarding Stem Completion priming in degenerative dementia is controversial. In a recent review of the literature, Fleischman et al. [14] pointed out only a slight prevalence in the number of studies detecting abnormal Stem Completion priming in patients with $\mathrm{AD}$ relative to studies documenting comparable priming in demented and age-matched normal controls. As a possible source of variance between studies reporting normal or abnormal priming in patients with dementia, these authors individuated the kind of orienting task used during the study phase. In particular, studies documenting abnormal priming in patients with $\mathrm{AD}$ most frequently requested subjects to read words or to rate pleasantness of words during the study phase. In contrast, the majority of studies that found normal Stem Completion priming in demented patients requested subjects to generate words or their meanings during the study phase. Accordingly, Fleischman et al. [14] identified the differential ability of the orienting tasks to induce semantic processing in patients with $\mathrm{AD}$ as the main reason for the discrepant priming levels in demented patients observed in various studies. In particular, just reading or pleasantness rating would produce insufficient processing in patients with $\mathrm{AD}$, thus resulting in poor priming. In contrast, generating the target words or their meaning would produce reliable processing also in demented patients, thus resulting in normal priming. Indeed, this explicative hypothesis is contradicted from opposite points of view both by Fleischman et al.'s [14] and our results. Fleischman et al. [14] documented normal priming in patients with $\mathrm{AD}$ both when the orienting task consisted of reading or generating the word. In contrast, we found deficient priming in demented patients both following pleasantness rating (Experiment 2) and meaning generation (semantic condition of Experiment 1).

As an alternative reason for the discrepant findings emerging from different studies, it is possible that patients with dementia participating in studies which documented abnormal priming in AD suffered from a more severe cognitive deterioration than patients sampled in studies showing comparable priming in $\mathrm{AD}$ and control groups. Also in this case, however, an examination of the literature and of our own results hardly supports this hypothesis. As documented by Fleischman et al. [14], severity of dementia is not a significant predictor of the priming level exhibited by patients with AD in different studies. As a confirmation of this, average Mini Mental Examination scores of patients participating in Experiments 1 and 2 of the present study were alternatively lower $[10,14]$ or higher $[12,40]$ than average Mini Mental Examination scores exhibited by AD samples which achieved normal levels of Stem Completion priming in previous studies. Moreover, in both experiments of the present study, the Stem Completion priming was abnormal also in a subsample of patients with $\mathrm{AD}$ affected by mild forms of mental impairment. 
In conclusion, the priming effect elicited by Stem Completion in various groups of patients with AD is not consistent. Reasons for such conflicting results are not clear. However, when compared to the high consistency of results documenting normal Stem Completion priming in anterograde amnesic patients, the bulk of the evidence no doubt suggests impaired Stem Completion priming in degenerative demented patients. By manipulating experimental conditions during study, we demonstrated that both lexical and conceptual components of the repetition priming elicited by the Stem Completion task were defective in patients with $\mathrm{AD}$.

If the dissociation between normal lexical and deficient conceptual repetition priming is insufficient to account for the abnormal priming displayed by patients with $\mathrm{AD}$ on the Stem Completion test, then why do these patients show normal priming in procedures such as Identification of tachistoscopically presented words and non-words [28,29], Fragmented Picture Identification [16] and Text Rereading [36] and, in contrast, deficient priming in tests such as Stem Completion, Free Association [4,43] or Category Exemplar production [35]? That is, which variable critically differentiates experimental procedures eliciting normal or deficient repetition priming in demented patients?

Monti et al. [36] proposed a number of other dimensions along which repetition priming tests can be distinguished, possibly providing a better theoretical basis for explaining dissociations between normal and abnormal priming in demented patients. For example, these authors proposed distinguishing between procedures giving rise to short-lived priming (e.g., Free Association, Stem Completion), resulting in deficient priming in patients with $\mathrm{AD}$, and procedures producing long-lived priming (e.g., Word Identification, Lexical Decision), generally raising normal priming in demented patients. However, as these authors pointed out, despite the repetition priming effect elicited by Text Rereading is short-lived [38], it is normal in AD patients. Another interpretative framework argues that $\mathrm{AD}$ patients suffer from a hyperspecificity of processing operations, preventing them from normally translating different operations during study and test phases [32]. This view posits a distinction between procedures in which the same tasks are performed during study and testing (e.g., Word or Picture Identification), providing normal priming in patients with $\mathrm{AD}$, and procedures based on different tasks during study and testing (e.g., Stem Completion), frequently impaired in patients with $\mathrm{AD}$. In contrast with this explicative hypothesis, we recently provided evidence of fully normal repetition priming elicited by Text Rereading in AD patients also when the experimental text had only been listened to during the study phase [7]. In our opinion, the dichotomy which actually provides the strongest explicative value is that between repetition priming tests requiring generation of the target starting from a fragment of the whole stimulus (e.g., Stem Completion or Free Association) and procedures simply requiring identification of perceptually degraded stimuli (e.g., Word or Picture Identification). Tests based on generation procedures (such as Stem Completion or Free Association) evoke deficient repetition priming in patients with AD. In contrast, tests that simply require reading (e.g., Word Identification, Text Rereading) or recognizing pictures (Fragmented Picture Identification) generally elicit normal facilitation in demented patients (see [16] for a similar interpretative hypothesis). Although a strong theoretical explanation of this kind of dissociation is lacking, we can temporarily conclude that repetition priming in patients with $\mathrm{AD}$ is impaired at a level that significantly reduces the advantage of previously studied stimuli being generated in response to a fragment cue over unstudied stimuli. However, the residual priming in these patients is still able to support an acceleration or an increased accuracy in the identification of previously processed material.

\section{References}

[1] Bassili JN, Smith MC, MacLeod CM. Auditory and visual Word-Stem Completion: Separating data-driven and conceptual-driven processes. Quarterly Journal of Experimental Psychology 1989;41A:439-53.

[2] Bowers JS, Schacter DL. Implicit memory and test awareness. Journal of Experimental Psychology: Learning, Memory, and Cognition 1990;16:404-16.

[3] Brown AS, Mitchell DB. A reevaluation of semantic versus nonsemantic processing in implicit memory. Memory and Cognition 1994;22:533-41.

[4] Carlesimo GA. Perceptual and conceptual priming in amnesic and alcoholic patients. Neuropsychologia 1994;32:903-21.

[5] Carlesimo GA, Fadda L, Marfia G, Caltagirone C. Explicit memory and repetition priming in dementia: evidence for a common basic mechanism underlying conscious and unconscious retrieval deficits. Journal of Clinical and Experimental Neuropsychology 1995;17:44-57.

[6] Carlesimo GA, Marfia GA, Loasses A, Caltagirone C. Perceptual and conceptual components in implicit and explicit Stem Completion: A neuropsychological investigation. Neuropsychologia 1996;34:785-92.

[7] Carlesimo GA, Mauri M, Fadda L, Turriziani P, Caltagirone C. Intact crossmodal text-specific repetition priming in patients with Alzheimer's disease (submitted for publication).

[8] Cermak LS, Talbot N, Chandler K, Wolbarst LR. The perceptual priming phenomenon in amnesia. Neuropsychologia 1985;23:615-22.

[9] Chiarello C, Hoyer WJ. Adult age differences in implicit and explicit memory: time course and encoding effects. Psychology and Aging 1988;3:358-66.

[10] Christensen H, Birrell P. Explicit and implicit memory in dementia and normal ageing. Psychological Research 1991;53:149-61.

[11] De Mauro T, Mancini F, Vedovelli M, Voghera M. Lessico di frequenza dell'italiano parlato. Milano: Etaslibri, 1993.

[12] Deweer B, Ergis AM, Fossati P, Pillon B, Boller F, Agid Y, 
Dubois B. Explicit memory, procedural learning and lexical priming in Alzheimer's disease. Cortex 1994;30:113-26.

[13] Fleischman DA, Gabrieli JDE, Reminger SL, Rinaldi JA, Wilson RS. Conceptual priming in perceptual identification for patients with Alzheimer's disease and a patient with right occipital lobectomy. Neuropsychology 1995;9:187-97.

[14] Fleischman DA, Gabrieli JDE, Rinaldi JA, Reminger SL, Grinnell ER, Lange KL, Shapiro R. Word-stem completion priming for perceptually and conceptually encoded words in patients with Alzheimer's disease. Neuropsychologia 1997;35:25-35.

[15] Folstein MF, Folstein SE, McHugh PR. 'Mini-mental state': A practical method for grading the cognitive state of patients for the clinician. Journal of Psychiatric Research 1975;12:189-98.

[16] Gabrieli JDE, Keane MM, Stanger BZ, Kjelgaard MM, Growdon JH. Dissociations among structural-perceptual, lexical-semantic, and event-fact memory systems in amnesic, Alzheimer's, and normal subjects. Cortex 1994;30:75-103.

[17] Graf P, Mandler G. Activation makes words more accessible, but not necessarily more retrievable. Journal of Verbal Learning and Verbal Behavior 1984;23:553-68.

[18] Graf P, Mandler G, Haden PE. Simulating amnesic symptoms in normal subjects. Science 1982;218:1243-4.

[19] Graf P, Shimamura AP, Squire LR. Priming across modalities and priming across category levels: extending the domain of preserved function in amnesia. Journal of Experimental Psychology: Learning, Memory, and Cognition 1985;11:386-96.

[20] Graf P, Squire LR, Mandler G. The information that amnesic patients do not forget. Journal of Experimental Psychology: Learning, Memory, and Cognition 1984;10:164-78.

[21] Hachinski VC, Iliff LD, Zihka E, DuBoulay GH, McAllister VL, Marshall J, Russel RWR, Symon L. Cerebral blood flow in dementia. Archives of Neurology 1975;32:632-7.

[22] Hamman SB, Squire LR. Level-of-processing effects in wordcompletion priming: A neuropsychological study. Journal of Experimental Psychology: Learning, Memory, and Cognition 1996;22:933-47.

[23] Heindel WC, Butters N, Salmon DP. Impaired learning of a motor skill in patients with Huntington's disease. Behavioural Neurosciences 1988;102:141-7.

[24] Huff FJ, Mack L, Mahlmann J, Greenberg S. A comparison of lexical-semantic impairments in left hemisphere stroke and Alzheimer's disease. Brain and Language 1988;34:262-78.

[25] Hughes CP, Berg L, Danziger WL, Coben LA, Martin RL. A new clinical scale for the staging of dementia. British Journal of Psychiatry 1982;140:566-72.

[26] Jacoby LL, Dallas M. On the relationship between autobiographical memory and perceptual learning. Journal of Experimental Psychology: General 1981;110:306-40.

[27] Java RI, Gardiner JM. Priming and aging: Further evidence of preserved memory function. American Journal of Psychology 1991;104:89-100.

[28] Keane MM, Gabrieli JDE, Fennema AC, Growdon JH, Corkin S. Evidence for a dissociation between perceptual and conceptual priming in Alzheimer's disease. Behavioural Neuroscience 1991;105:326-42.

[29] Keane MM, Gabrieli JDE, Growdon JH, Corkin S. Priming in perceptual identification of pseudowords is normal in Alzheimer's disease. Neuropsychologia 1994;32:343-56.

[30] Kirsner K, Milech D, Stander P. Common and modality-specific processes in the mental lexicon. Memory and Cognition 1983;11:621-30.

[31] Lupker SJ, Harbluk JL, Patrick AS. Memory for things forgotten. Journal of Experimental Psychology: Learning, Memory, and Cognition 1991;17:897-907.

[32] Maki PM, Knopman DS. Limitations of the distinction between conceptual and perceptual implicit memory: A study of Alzheimer's disease. Neuropsychology 1996;10:464-74.

[33] McKhann G, Drachman D, Folstein M, Katzman R, Price D, Stadlan EM. Clinical diagnosis of Alzheimer's disease: Report of the NINCDS-ADRDA work group under the auspices of Department of Health and Human Services Task Force on Alzheimer's disease. Neurology 1984;34:939-44.

[34] Micco A, Masson MEJ. Implicit memory for new associations: An interactive process approach. Journal of Experimental Psychology: Learning, Memory, and Cognition 1991;17:110523.

[35] Monti LA, Gabrieli JDE, Reminger SL, Rinaldi JA, Wilson RS, Fleischman DA. Differential effects of aging and Alzheimer's disease on conceptual implicit and explicit memory. Neuropsychology 1996;10:101-12.

[36] Monti LA, Gabrieli JDE, Wilson RS, Reminger SL. Intact textspecific implicit memory in patients with Alzheimer's disease. Psychology and Aging 1994;9:64-71.

[37] Morrison JH, Hof P, Campbell MJ, De Lima AD, Voigt T, Bouras C, Cox K, Young WG. Cellular pathology in Alzheimer's disease: Implications for corticocortical disconnection and differential vulnerability. In: Rapoport SI, Petit H, Leys D, Christen Y, editors. Imaging, cerebral topography and Alzheimer's disease. Berlin: Springer, 1990. p. 19-40.

[38] Musen G, Shimamura AP, Squire LR. Intact text-specific reading skill in amnesia. Journal of Experimental Psychology: Learning, Memory, and Cognition 1990;16:1068-76.

[39] Nebes RD. Semantic memory in Alzheimer's disease. Psychological Bulletin 1989;106:377-94.

[40] Partridge FM, Knight RG, Feehan M. Direct and indirect memory performance in patients with senile dementia. Psychological Medicine 1990;20:111-8.

[41] Richardson-Klavehn A, Gardiner JM. Depth-of-processing effects on priming in Stem Completion: Tests of the voluntarycontamination, conceptual processing, and lexical-processing hypothesis. Journal of Experimental Psychology: Learning, Memory, and Cognition 1998;24:593-609.

[42] Roediger HL. Implicit memory retention without remembering. American Psychologist 1990;45:1043-56.

[43] Salmon DP, Shimamura AP, Butters N, Smith S. Lexical and semantic priming deficits in patients with Alzheimer's disease. Journal of Clinical and Experimental Neuropsychology 1988;10:477-94.

[44] Shimamura AP, Salmon DP, Squire LR, Butters N. Memory dysfunction and word priming in dementia and amnesia. Behavioural Neuroscience 1987;101:347-51.

[45] Shimamura AP, Squire LR. Paired-associate learning and priming effects in amnesia: a neuropsychological study. Journal of Experimental Psychology: General 1984;113:556-70.

[46] Smith ME, Oscar-Berman M. Repetition priming of words and pseudowords in divided attention and in amnesia. Journal of Experimental Psychology: Learning, Memory, and Cognition 1990;6:1033-42.

[47] Tulving E, Schacter DL. Priming and human memory systems. Science 1990;247:301-6.

[48] Weldon MS. Mechanisms underlying priming on perceptual tests. Journal of Experimental Psychology: Learning, Memory, and Cognition 1991;17:526-41.

[49] Wilson BA, Green R, Teasdale T, Beckers K, Della Sala S, Kaschel R, Schuri U, Van der Linden M, Weber E. Implicit learning in amnesic subjects: A comparison with a large group of normal control subjects. The Clinical Neuropsychologist 1996;10:279-92.

[50] Zingarelli N. Vocabolario della lingua italiana. Milano: Zanichelli, 1983. 